\title{
A Case of Infected Left Atrial Myxoma With Concomitant Mitral Valve Endocarditis
}

\author{
Jae-Hee Chang, $\mathrm{MD}^{1}$, Jeong-Yeon Kim, $\mathrm{MD}^{2}$, Jin-Won Yoon, $\mathrm{MD}^{1}$, Myung-Do Seol, $\mathrm{MD}^{1}$, \\ Dong-Jun Won, $\mathrm{MD}^{1}$, Wook-Hyun Cho, $\mathrm{MD}^{1}$, Jae-Jin Lee, $\mathrm{MD}^{3}$, and Hye-Jae Jo, $\mathrm{MD}^{4}$ \\ ${ }^{1}$ Division of Cardiology and ${ }^{2}$ Infection, Departments of Internal Medicine, ${ }^{3}$ Cardiac Surgery and ${ }^{4}$ Pathology, \\ Samyook Medical Center, Seoul, Korea
}

\section{ABSTRACT}

Myxoma is the most common primary tumor in the heart. Cardiac myxomas can present in various manners including embolization and fever, sometimes simulating endocarditis. However, they are rarely infected. We report here a case of an infected left atrial myxoma that seeded a normal mitral valve and atypically presented with multiple embolic events in the lower extremities along with multiple splenic and a cerebellar infarction. (Korean Circ J 2011;41:618-621)

KEY WORDS: Myxoma; Endocarditis.

\section{Introduction}

Cardiac myxomas can present in various ways, ${ }^{12)}$ and in rare cases they are infected. Infected cardiac myxoma with concomitant infective endocarditis is very rare, and not previously reported in Korea. Here, we report a case of 49 -yearold male patient who was initially diagnosed with infected left atrial myxoma with atypical presentation of metastatic infection of the lower leg, that was later found to be complicated by concomitant infective endocarditis of the mitral valve during surgery. He was successfully treated with antibiotic therapy and mitral valve replacement.

\section{Case}

A 49-year-old previously healthy male was admitted to the

Received: November 12, 2010

Revision Received: December 22, 2010

Accepted: January 10, 2011

Correspondence: Wook-Hyun Cho, MD, Division of Cardiology, Department of Internal Medicine, Samyook Medical Center, 29-1 Hwigyeong 2-dong, Dongdaemun-gu, Seoul 130-092, Korea

Tel: 82-2-2210-3250, Fax: 82-2-2212-2673

E-mail: mulgang@gmail.com

- The authors have no financial conflicts of interest.

(a) This is an Open Access article distributed under the terms of the Creative Commons Attribution Non-Commercial License (http://creativecommons.org/licenses/by-nc/3.0) which permits unrestricted non-commercial use, distribution, and reproduction in any medium, provided the original work is properly cited. hospital with fever and pain in the left calf. Two months before admission, the patient had fever, chills and myalgias, especially in the leg. He visited a local clinic and had taken medication for a total of three weeks. However the fever, which was remittent, did not completely resolve. A few weeks before admission, he had painful and erythematous lesions in both thighs, which gradually disappeared. One week before admission, he developed pain in the left calf and a headache. He denied overseas travel, sick exposures, or history of trauma.

Upon physical examination, the patient's blood pressure was $90 / 60 \mathrm{~mm} \mathrm{Hg}$, pulse was 80 beats per minute, an temperature was $38^{\circ} \mathrm{C}$. He had periodontitis and dental caries. The first and second heart sounds were normal and no heart murmurs were heard. No lymphadenopathy or hepatosplenomegaly was noted. The patient's left calf was slightly erythematous, swollen and tender. His lower extremities were otherwise normal, and the remainder of the examination was unremarkable.

Laboratory values upon admission were as follows: white blood cell count, $11,700 / \mathrm{mm}^{3}$ with $70.3 \%$ neutrophils; hemoglobin, $13.7 \mathrm{~g} / \mathrm{dL}$; platelet count, $254,000 / \mathrm{mm}^{3}$; blood urea nitrogen, $8.1 \mathrm{mg} / \mathrm{dL}$; serum creatinine, $1.0 \mathrm{mg} / \mathrm{dL}$; glucose, 136 $\mathrm{mg} / \mathrm{dL}$; alanine aminotransferase, $16 \mathrm{U} / \mathrm{L}$; aspartate aminotransferase, $29 \mathrm{U} / \mathrm{L} ; \mathrm{C}$-reactive protein, $7.91 \mathrm{mg} / \mathrm{dL}$. Urinalysis showed no abnormalities. Chest X-ray and electrocardiography were unremarkable.

On the third hospital day, magnetic resonance imaging (MRI) of his left calf revealed inflammation in the medial 
belly of the gastrocnemius muscle without abscess (Fig. 1). Empiric antimicrobial therapy for myositis was started with ceftriaxone and clindamycin intravenously. Considering the unusual location of inflammation and his history of no previous trauma, metastatic infection from an unrecognized primary site was also considered. On the sixth day of admission, a small, palpable, tender erythematous lesion was observed on the left thumb, suggestive of an Osler's node. Transthoracic echocardiography and transesophageal echocardiogra-

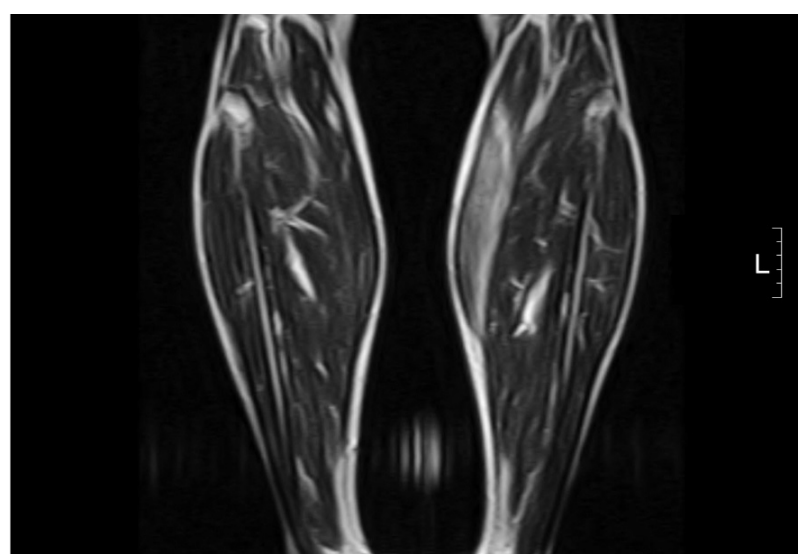

Fig. 1. T2-weighted image of the left gastrocnemius. This image shows an abnormally ill defined high signal that suggests inflammation in the medial belly of the left gastrocnemius. phy were then performed, which revealed normal sized cardiac chambers and normal systolic function of the left ventricle. All cardiac valves were clean and only trivial mitral regurgitation was noted. An elongated, villous, $5.6 \times 1.8 \mathrm{~cm}$ mass arising from the posterior wall of the left atrium (Fig. 2) was found. The initial blood cultures drawn at the time of admission yielded Streptococcus bovis resistant to gentamicin. A diagnosis of infected left atrial myxoma was made and antimicrobial therapy was continued. On the seventh hospital day the patient complained of continuing headache and noncontrast brain magnetic resonance imaging incidentally showed an infarction of the left cerebellar hemisphere that was attributed to embolization from the infected myxoma. On the tenth hospital day an abdominal CT scan was conducted to check for additional foci of infection, which revealed multiple splenic infarcts. After twelve days of antibiotics, the patient's fever had resolved and after 19 days of antibiotics the patient underwent excision of the mass.

In the operating room, the mass was found to have a base of about $9 \mathrm{~mm}$ (Fig. 3A) and was attached to the posterior wall of the left atrium. Unexpectedly, multiple tiny and nodular vegetations of less than $1 \mathrm{~mm}$ in size were found on the endocardium of the left atrium and P2 and P3 portion of the posterior mitral leaflet (Fig. 3B) and posterior mitral annu-
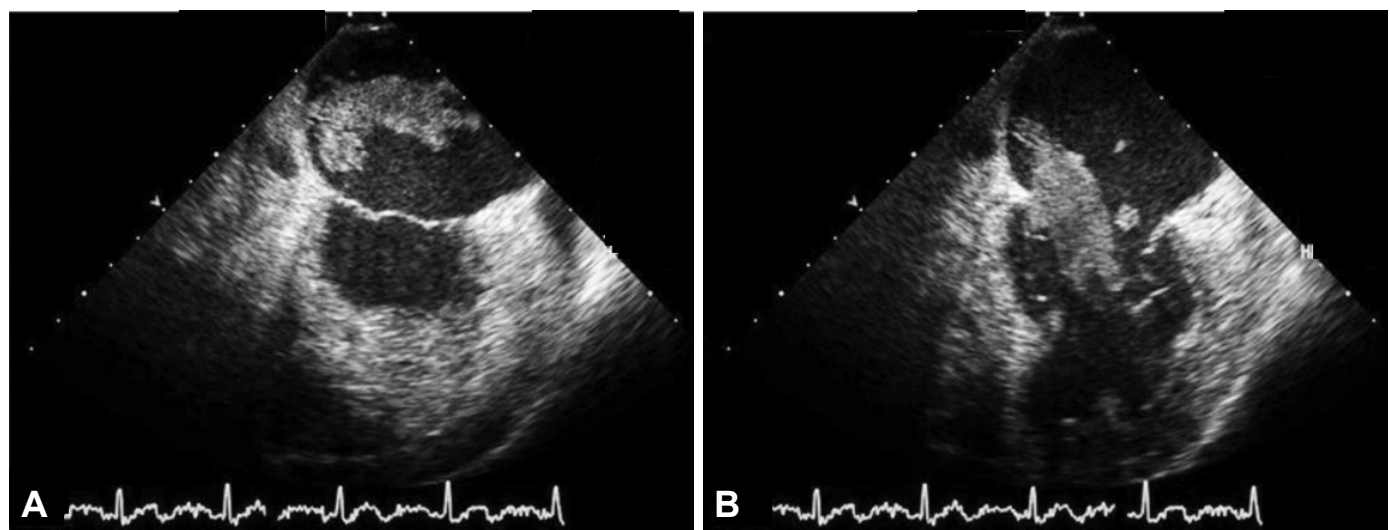

Fig. 2. Transesophageal echocardiography. A: echocardiography shows a left atrial tumor of $5.6 \times 1.8 \mathrm{~cm}$ with a villous surface in the systole. $B$ : the mass protrudes from the left atrium into the left ventricle in the diastole.
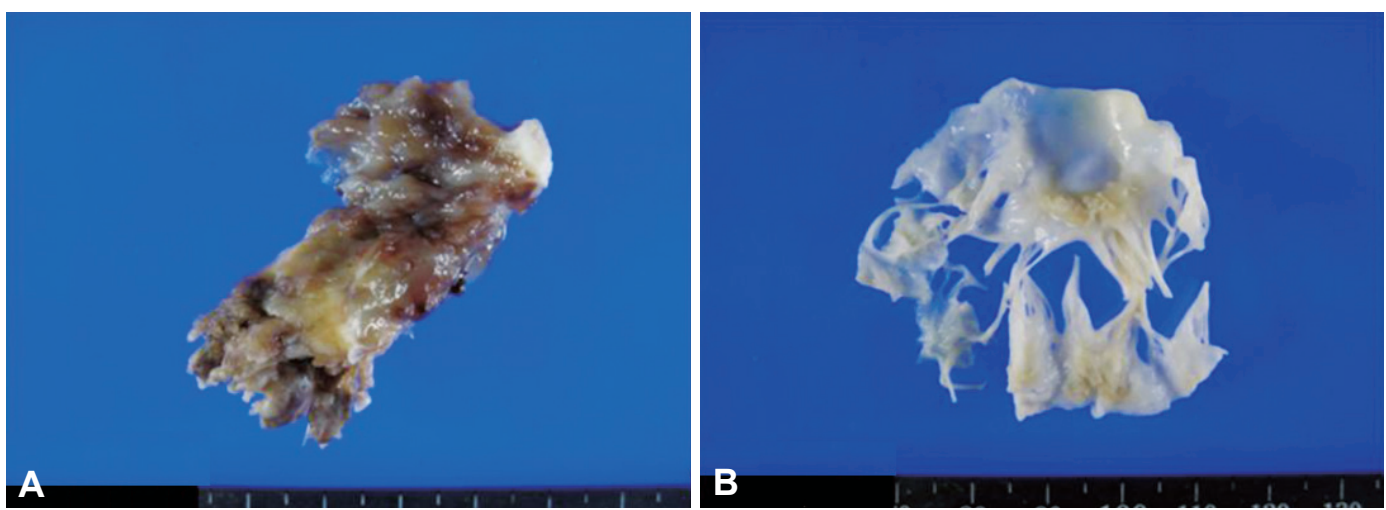

Fig. 3. Gross specimen of the mass and the mitral valve. A: the mass has a villous surface with a base of about $9 \mathrm{~mm}$. B: multiple tiny and nodular vegetations of less than $1 \mathrm{~mm}$ are found on the P2 and P3 portion of the posterior mitral leaflet. 


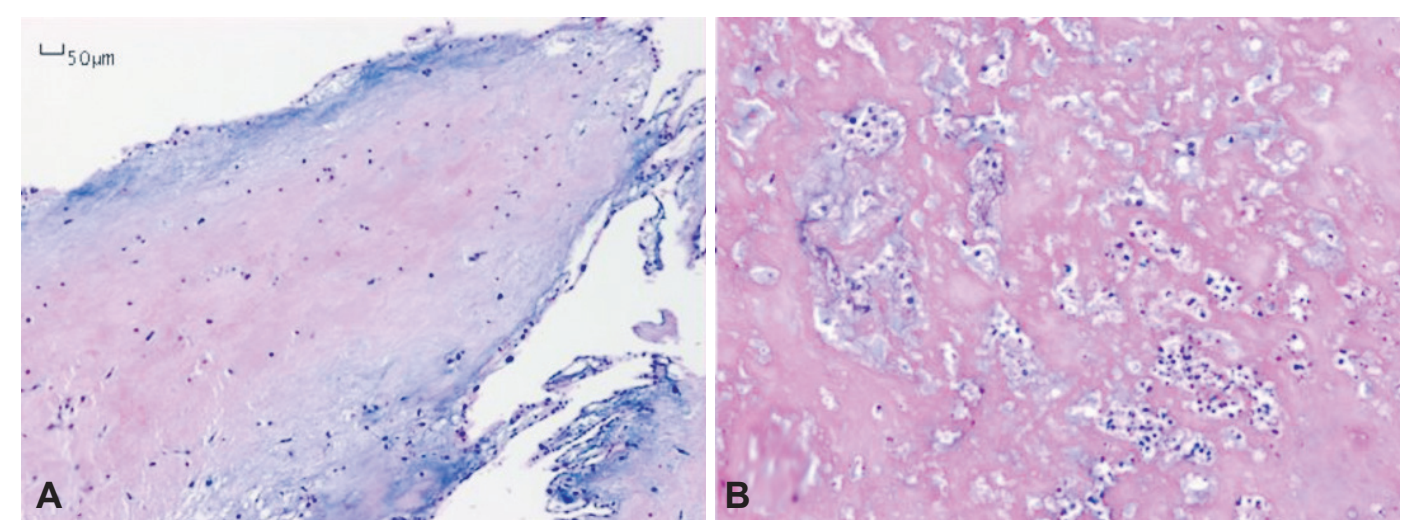

Fig. 4. Microscopic features of the specimen. A: the mass is composed of small round polygonal cells in the loose myxoid stroma (Hematoxylin and Eosin staining, 40× magnification). B: higher magnification of the superficial lesion reveals aggregates of polymorphonuclear neutrophils in degenerating stroma, suggesting microabscess (Hematoxylin and Eosin staining, 200× magnification).

lus. Since they were too firmly attached to be removed without mitral valve injury, the mass was resected and mitral valve replacement was conducted using a Saint-Jude $29 \mathrm{~mm}$ prosthetic valve.

The pathology of the excised mass showed a partially myxoid and papillomatous surface and microscopically ischemic necrosis and focal abscesses (Fig. 4). Focal microabscesses and multinodular vegetations with sizes of less than $1 \mathrm{~mm}$ were present on the mitral valve, which was consistent with subchronic endocarditis.

The patient rapidly recovered without postoperative complications and was discharged 12 days after the operation. Three sets of blood cultures drawn during the perioperative period yielded no growth of any organisms.

\section{Discussion}

Cardiac myxomas can present in various ways ${ }^{12)}$ and are infected in rare cases. ${ }^{3 / 4)}$ The clinical features of endocarditis and an infected cardiac myxoma are virtually identical. A case of infected cardiac myxoma with infective endocarditis concurrently is very rare, and not previously reported in Korea. Cardiac myxoma and infective endocarditis can induce embolism. The incidence of cerebral or systemic embolization occurring from an infected myxoma is two to three-fold higher than that of non-infected myxoma. ${ }^{4) 5}$ Our patient suffered from migrating myositis, splenic infarction and a left cerebellar infarction as a consequence of emboli.

Echocardiography is useful during diagnosis of myxoma or endocarditis. The presence of a finger-like structure on the surface of a myxoma may establish the diagnosis of infection upon transesophageal echocardiography. ${ }^{6}$ The morphologic finding of the myxoma in this case demonstrated a villous surface resembling a finger-like structure. Taken together, the echocardiographic findings and clinical features of systemic infected embolism suggested an infected myxoma.

Although transesophageal echocardiography is highly sen- sitive for valvular vegetations, it has a negative predictive value of $87.8 \%$ and rare cases may be missed.") In this case, mitral valve endocarditis was not observed upon preoperative evaluations, presumably because the size of the vegetations on the endocardium and mitral valve were too small to be demonstrated on transesophageal echocardiography. Thus, endocarditis may be concomitantly associated with infective myxo$\mathrm{ma}$, even if no findings of endocarditis are observed upon transesophageal echocardiography.

The pathologic findings revealed that the sizes of the abscesses were larger in the myxoma than the mitral valve and tiny vegetations were scattered in the endocardium of the left atrium and focal areas of the mitral valve. Therefore, we speculated that bacteremia initially infected the myxoma, and secondarily infected the mitral valve.

The pathogen in this case was Streptococcus bovis, which is known to be the causative agent in 5 to $14 \%$ of the cases of endocarditis. ${ }^{8}$ Streptococcus bovis is reported to be related to colorectal neoplasms in elderly patients through unknown mechanisms." Because the history and symptoms of the patient were nonspecific for gastrointestinal abnormalities, and the abdominal CT scan showed no sign of colonic wall thickening or obstruction, further evaluation including colonoscopy was not performed.

The correct treatment strategy for infected atrial myxoma has yet to be settled. ${ }^{10) 111}$ However, in light of the low operative risk and good long-term prognosis of myxoma resection, surgery should be conducted urgently to avoid complications. However, in the present case, infected endocarditis and systemic embolism, such as cerebellar infarction, splenic infarction, and septic emboli in the lower extremities, were followed by infective myxoma. When infective endocarditis and/ or infected myxoma is complicated with embolic infarction, the timing of the operation must be carefully determined, taking into consideration of the risk of additional embolization, the size and nature of cerebral infarction, and the sensitivity of antibiotics against the pathogen. Eishi et al. reported 
that cerebral damage was exacerbated postoperatively in $43.8 \%$ of the patients with infective endocarditis who underwent a cardiac operation within seven days of cerebral infarction. However, if the delay is more than two weeks, the exacerbation rate is around $10 \%{ }^{12)}$ Complicated cases of combined infected myxoma and infective endocarditis can also be guided according to Eishi's result.

Here, we report a case of infected left atrial myxoma and concomitant infective endocarditis of the mitral valve. After diagnosis of infected myxoma is established, surgery should be conducted promptly because of the possibility of embolic complications or infective endocarditis.

\section{REFERENCES}

1) Rhim HY, Youn HJ, Park JW, et al. Clinical experience of cardiac myxoma. Korean Circ J 1999;29:1317-23.

2) Kim CJ, Doh MH, Kwon OH, et al. Clinical observation of cardiac myxoma. Korean Circ J 1985;15:671-9.

3) Llinares P, Mlguue E, Echamz A, Juffé A, Prado G, Alvarez N. Infected atrial myxoma simulating infective endocarditis. Enferm Infecc Microbiol Clin 1993;11:378-81.
4) Whitman MS, Rovito MA, Klions D, Tunkel AR. Infected atrial myxoma: case report and review. Clin Infect Dis 1994;18:657-8.

5) Uchino K, Mochida Y, Ebina T, et al. Infected left atrial myxoma. Intern Med 2002;41:957-60.

6) Tunick PA, Fox AC, Culliford A, Levy R, Kronzon I. The echocardiographic recognition of an atrial myxoma vegetation. Am Heart $J$ 1990;119:679-80.

7) Law A, Honos G, Huynh T. Negative predictive value of multiplane transesophageal echocardiography in the diagnosis of infective endocarditis. Eur J Echocardiogr 2004;5:416-21.

8) Duval X, Papastamopoulos V, Longuet P, et al. Definite Streptococcus bovis endocarditis: characteristics in 20 patients. Clin Microbiol Infect 2001;7:3-10.

9) Klein RS, Recco RA, Catalano MT, Edberg SC, Casey JI, Stigbigel $\mathrm{NH}$. Association of Streptococcus bovis with carcinoma of the colon. N Engl J Med 1977;297:800-2.

10) Dekkers P, Elbers HR, Morshuis WJ, Jaarsma W. Infected left atrial myxoma. J Am Soc Echocardiogr 2001; 14:644-5.

11) Kim H, Kang JK, Chung YS, Kim YH, Chung WS. Infected left atrial myxoma. Korean J Thorac Cardiovasc Surg 2009;42:513-5.

12) Eishi K, Kawazoe K, Kuriyama Y, Kitoh Y, Kawashima Y, Omae T. Surgical management of infective endocarditis associated with cerebral complications. Multi-center retrospective study in Japan. J Thorac Cardiovasc Surg 1995;110:1745-55. 